\title{
RELATIONSHIP BETWEEN CZECH PARENT AND CHILD PEDOMETER-ASSESSED WEEKDAY AND WEEKEND PHYSICAL ACTIVITY AND SCREEN TIME
}

\author{
Erik Sigmund, Dagmar Sigmundová, Petr Bad'ura, Jaroslava Voráčová \\ Institute of Active Lifestyle, Faculty of Physical Culture, Palacký University Olomouc, Olomouc, Czech Republic
}

\section{SUMMARY}

Aim: Uncovering the influences of parents' behaviour on their children's physical activity provides an insight into the lifestyle of families and development of effective family-based interventions. The aim of this study was to determine the relationship between parents' behaviour (step count (SC) and screen time (ST)) and children's SC on weekdays and at weekends.

Methods: The participants (388 parents aged 35-45 and their 485 children aged 9-12) were randomly recruited from 21 Czech governmentfunded primary schools. The participants recorded SC and ST duration for seven consecutive days ( $\geq 10 \mathrm{~h} /$ day) during April-May and September-October 2013. The associations between parents' behaviour (SC and ST) and children's SC were estimated using general linear regression separately for weekdays and weekends. Each 1,000 SC increase in mothers' (fathers') SC/weekday was associated with an extra $261 \mathrm{SC} /$ day in their daughters and 413 (244) SC/day in their sons.

Results: Each 1,000 SC increase in mothers' (fathers') SC/weekend day was associated with an extra 523 (386) SC/day in their daughters and 508 (435) SC/day in their sons. A reduction in mothers' ST by 30 minutes per weekend day was associated with an extra 494 SC/day in their daughters and $467 \mathrm{SC} /$ day in their sons.

Conclusions: This study reveals a quantifiable relationship between parent-child SC/day and mothers' ST and children's SC at weekends. Weekend days are more suitable for the implementation of family-based interventions.

Key words: step count, screen time, Yamax pedometer, daughters and sons, weekdays and weekends

Address for correspondence: E. Sigmund, Faculty of Physical Culture, Palacký University Olomouc, trída Míru 117, 77111 Olomouc, Czech Republic. E-mail: erik.sigmund@upol.cz

\section{INTRODUCTION}

The prevalence of childhood obesity has risen substantially in the long-term (thirty years), medium-term (eight years) and shortterm (two years) perspective in developed as well as developing countries (1-3). In addition, it is alarming that in developing countries the peak prevalence of obesity has moved from older adults to younger age cohorts (1). Although childhood obesity is the result of a complex interaction among multiple behavioural, biological and environmental factors that adversely affect longterm energy balance (4), lifestyle factors are also believed to contribute to weight gain and risk of obesity in youth $(5,6)$. This provides space to investigate the role of parents in shaping the energy-balanced behaviour of their children (7-11).

Although global efforts have failed to reduce the prevalence of childhood obesity, there are effective school-based (12-16), community-based (17) and family-based (18) interventions aimed at reducing overweight and obesity in children. The current metaanalyses are consistent with the conclusion that the active participation of parents in nutrition and physical activity interventions is more effective in maintaining the healthy body weight of their children than interventions without active parental involvement (18-20). However, parental involvement in child intervention programmes is multifaceted, including, for example, lessons for participating parents dealing with healthy nutrition principles, physical activity and sedentary behaviour; family group sessions and consultations; application of parenting styles; and joint physical activities among parents and children $(15,17,19,20)$. An important part of effective interventions aimed at reducing overweight and obesity, as well as promoting development in childhood, is regular realisation of health-enhancing physical activity (PA) $(6,12-15,17)$.

A well-documented positive relationship exists between objectively measured PA in parents and their children $(7-9,11,21-24)$, and parent-child self-reported screen time $(25,26)$. However, there are considerable differences in the parent-child PA relationship (parent-child screen time relationship) between weekdays/school days and weekends $(9,11,23,24,26)$ and between parents' and children's gender $(8,9,24,26)$. At weekends, research repeatedly reveals lower levels of PA and increased sedentary behaviour for both children and parents in comparison with weekdays/school days $(9,23,24,26)$. Weekend days still represent a 'critical window' for children and parents to accumulate necessary healthrelated PA $(9,12,15,24,27)$.

The results of a substudy within the Canadian Physical Activity Levels among Youth (CANPLAY) surveillance study highlighted that every increase by 1,000 steps per day for mothers (fathers) is associated with 195-219 (273) extra steps per day in their daughters and 263-439 (329-407) extra steps per day in their sons (8). Another population-based study, the Raising Healthy 
Eating and Active Living Kids in Alberta (REAL Kids Alberta) study, revealed that girls whose parents engaged in PA with them more than 4 times per week achieved an additional 890 steps per school day compared with girls whose parents engaged in joint PA 1-3 times per week (23). Furthermore, if parents, regardless of gender, spent more than 2 hours per day watching television (TV), children were at least 3.4 times more likely to spend more than 2 hours watching TV on weekdays (26). Even at weekends, daughters (sons) were 7.9 (3.8) times more likely to exceed the 2 hours of watching TV if their fathers spent more than 2 hours watching TV (26). Therefore, increasing children's PA level and reducing ST, particularly at weekends, is still a priority for improving child health outcomes (27).

Although a quantifiable relationship between objectively measured PA of parents and their children is documented, it is not fully understood how this relationship varies during weekdays/school days and weekends $(8,9,24)$. Another unclear area is the relationship between the level of parents' screen time and children's PA and its changes during weekdays/school days and weekends. Given the high prevalence of childhood overweight and obesity in Central and Eastern European countries (1-3), and the absence of most of these countries in major international and European studies using objective measurement of children's PA $(4,10,28)$, it is desirable to analyse the patterns of parent-child PA and sedentary behaviour in Central and Eastern European countries as well.

Therefore, this study aims to address these shortcomings by examining the relationship between parents' behaviour (step counts and screen time) and children's step counts on weekdays and weekend days, and to provide practical information about the direct impact of parents' behaviour on their children's physical activity. The specific objectives were to:

- describe and compare gender-related differences in step counts and duration of screen time in parents and their children on weekdays and at weekends;
- quantify the association between parents' step counts/screen time and children's step counts on weekdays and at weekends.

\section{MATERIALS AND METHODS}

\section{Sample}

The data come from the New Technologies and Approaches to Physical Activity Monitoring: Utilization in Kinanthropology (NTAPAMUK) Project, which examines the influence of parents on the physical activity and sedentary time of 9-12 year old children from the Czech Republic. The participants were randomly recruited from 21 government-funded primary schools, all of which agreed to participate in this study. The selection of primary schools corresponded to the distribution of the urban-rural population in the Czech Republic (29). There were initially a total of 1,375 participants ( 720 children and 655 parents) - in 7 of the 14 regions in the Czech Republic: Vysočina Region, Moravia-Silesia Region, Olomouc Region, Pardubice Region, South Bohemia Region, South Moravia Region, and Zlín Region. The parents of 73.06\% of children ( $72.04 \%$ of daughters and $74.14 \%$ of sons) from the 4 th and 5 th grades of primary schools gave informed consent to participate in this study (Table 1). All participating girls and boys followed a mandatory daily school routine, as did the parents in their jobs during the weekdays of the monitored week. The data were collected during April-May and September-October 2013 under comparable daily climate conditions.

A total of 526 children (268 daughters and 258 sons) and their parents (252 mothers and 156 fathers) started the 8-day pedometer-based assessment of step counts and logbook recording of sedentary behaviour during the morning hours at school (children) and during the afternoon at home (parents). The measurement on the first day was excluded from the data analysis because the recording on the first day was incomplete and the novelty of wear-

Table 1. Descriptive characteristics (number, percentages, means and standard deviations) by gender

\begin{tabular}{|c|c|c|c|c|}
\hline & \multicolumn{2}{|c|}{ Parents } & \multicolumn{2}{|c|}{ Children } \\
\hline & Mothers & Fathers & Daughters & Sons \\
\hline Number $(\%)$ of selected respondents & $354(100 \%)$ & $301(100 \%)$ & $372(100 \%)$ & $348(100 \%)$ \\
\hline $\begin{array}{l}\text { Number (\%) of participants with informed } \\
\text { consent/pedometers issued }\end{array}$ & $252(71.19 \%)$ & $156(51.83 \%)$ & $268(72.04 \%)$ & $258(74.14 \%)$ \\
\hline $\begin{array}{l}\text { Number }(\%) \text { of returned pedometers meeting validation } \\
\text { criteria }\end{array}$ & $245(69.21 \%)$ & $143(47.51 \%)$ & $248(66.67 \%)$ & $237(68.10 \%)$ \\
\hline $\begin{array}{l}\text { Anthropometric variables of participants with valid } \\
\text { pedometer data }\end{array}$ & $(n=245)$ & $(n=143)$ & $(n=248)$ & $(n=237)$ \\
\hline Age (years) & $38.71 \pm 4.13$ & $41.48 \pm 5.58$ & $10.44 \pm 1.33$ & $10.57 \pm 1.26$ \\
\hline Body height (cm) & $166.12 \pm 13.85$ & $180.06 \pm 16.91$ & $144.43 \pm 9.72$ & $145.67 \pm 9.05$ \\
\hline Body weight (kg) & $67.04 \pm 11.35$ & $87.04 \pm 13.90$ & $36.87 \pm 9.19$ & $38.77 \pm 9.21$ \\
\hline $\mathrm{BMI}\left(\mathrm{kg} / \mathrm{m}^{2}\right)$ & $24.15 \pm 3.88$ & $26.61 \pm 2.84$ & $17.48 \pm 3.03$ & $18.11 \pm 3.11$ \\
\hline Overweighta, c & $24.68 \%$ & $56.43 \%$ & $12.05 \%$ & $15.74 \%$ \\
\hline Obesity ${ }^{b, d}$ & $7.66 \%$ & $12.14 \%$ & $6.43 \%$ & $12.34 \%$ \\
\hline
\end{tabular}

$\mathrm{n}$ - number of participants, BMI - body mass index; ${ }^{a}$ Overweight or bobesity in children represents a BMI from 85th to 97 th or greater than 97 th percentile of World Health Organisation growth charts (37); ' Overweight or dObesity in parents represents a Body Mass Index from $25 \mathrm{~kg} / \mathrm{m}^{2}$ to $29.9 \mathrm{~kg} / \mathrm{m}^{2}$ or greater than or equal to $30 \mathrm{~kg} / \mathrm{m}^{2}(38)$. 
ing the Yamax pedometer could have affected the initial activity (30). The data analysis included only records when the pedometer was worn for at least $10 \mathrm{~h}$ a day during at least 4 weekdays/school days and 2 weekend days. Monitoring of at least 4 weekdays and 2 weekend days is suitable for predicting weekly physical activity in children and young adults $(31,32)$.

Incomplete records of daily step counts or an omission of the age, body height and body weight variables constituted a reason for excluding $5.37 \%(n=20)$ of daughters, $6.04 \%(n=21)$ of sons, $1.97 \%(n=7)$ of mothers, and $4.3 \%(n=13)$ of fathers (Table 1$)$. The final sample with valid data on 7-day pedometer-derived step counts and logbook-recorded sedentary behaviour consisted of 485 children (248 girls and 237 boys) and 388 parents (245 mothers and 143 fathers).

\section{Instruments and Measurements}

The step counts in all participants were monitored using the same type of pedometer - the Yamax Digiwalker SW-200 (Yamax Corporation, Tokyo, Japan) - and a personalised individual logbook (32) for at least 10 continuous hours a day over 7 consecutive days.

The Yamax Digiwalker SW-200 is a commercially available, small and light $(1.5 \mathrm{~cm} \times 3.5 \mathrm{~cm} \times 5.0 \mathrm{~cm} ; 20 \mathrm{~g})$ electronic pedometer designed for measuring vertical oscillations. Each vertical oscillation exceeding the device threshold ( $\# 0.35 \mathrm{~g}$ ) is considered a step (33). Overall step counts, as the most accurate variable representing PA from the pedometer (34), are shown on the display of the device. Step counts assessed by the Yamax Digiwalker SW200 were validated against energy expenditure based on oxygen consumption $\left(\mathrm{VO}_{2}\right)$ in 9-12 year old boys and girls during walking and physical activity games $\left(\mathrm{r}_{\mathrm{s}}=0.78-0.92, \mathrm{p}<0.001\right)(32)$. The validity of free-living step counts of the Yamax pedometer was verified by a comparison with the ActiGraph GT1M in a 7-day monitoring (including both weekend days) of adolescent girls (35).

The personalised individual logbook consisted of 2 sections completed by children/parents: one for completing the step counts and the other for recording the duration of sedentary behaviour. The first section of the personalised individual logbook included the chronological structure of the day according to the current school schedule (paid employment for parents) to record the time and value shown on the display (step counts) of the Yamax pedometer 4 times a day (morning after wake-up, together with parent; start and end of school (paid employment for parents), together with teacher; evening before going to bed, together with parent). The second part of the logbook concerning sedentary behaviour comprised 7 items: sitting and lying while watching TV (DVD, video); sitting and lying down in front of a PC (notebook, tablet, smartphone); sitting or lying when studying, reading and playing (non-PC games, musical instruments, drawing and painting); sitting in a park or restaurant; sitting in a theatre or at a concert; sitting in a vehicle (car, bus, train, tram) and sitting in school (paid employment for parents). Children completed the second part of the logbook together with their parents in the evening. The accuracy of recording the duration of each type of sedentary behaviour was fixed at $10 \mathrm{~min}$. The daily duration of sedentary behaviour recorded in the logbook was validated against the daily duration of sedentary behaviour $(<100$ counts per min (cpm)) from the Actigraph accelerometer in 9-12 year old children (boys $-r_{s}=0.76$ and girls $-r_{s}=0.81, p<0.001$ ) (32).

On the first day of PA monitoring each child was provided with the Yamax pedometer with a small retaining strap for attachment to clothing, a pencil and a personalised individual logbook. The Yamax pedometer was not reset throughout the day. The children were instructed to wear the pedometer on the right hip, all day, for at least 10 hours a day, except for sleeping, personal hygiene and bathing (32). The children and teachers were instructed and trained to check, during the monitoring periods, correct attachment of the pedometer and correct reading and recording of the pedometer display data into the personalised individual logbook. The daily wearing time was computed as the difference between the morning (pedometer turned on) and evening time (pedometer turned off). The children also received pedometers and personalised individual logbooks for their parents.

The anthropometric characteristics of the participants were determined in advance before PA monitoring to prepare an individual logbook for each participant. One week before the start of the monitoring, parents were asked to provide information about their own body height and weight as well as the body height and weight of their children with $0.5 \mathrm{~cm}$ and $0.1 \mathrm{~kg}$ accuracy. The body height and body weight values of the family members participating in the survey were recorded by parents on the written informed consent form. The body height and body weight of children proxy-reported by their own parents/guardians and the derived BMI were validated against the direct measurement of body height (portable rigid stadiometer) and body weight (weight scale model: TBF 410, Tanita Corp., Tokyo, Japan) in 6-18 year old children $(\mathrm{ICC}=0.93-0.99, \mathrm{p}<0.001)(36)$.

\section{Statistical Analysis}

The data were analysed using the SPSS v.22 software (IBM SPSS, Inc., Chicago, IL, USA) and STATISTICA v.12 (StatSoft, Prague, Czech Republic).

The chronological age is represented by the difference between the date of birth and the first day of pedometer-based assessment of step counts and logbook-recorded sedentary behaviour. BMI $\left(\mathrm{kg} / \mathrm{m}^{2}\right)$ was calculated as body weight $(\mathrm{kg})$ divided by body height (m) squared. Obese, overweight and normal body mass in children were classified using the World Health Organisation (WHO) Child Growth Standards for girls and boys between the ages of 5 and 19 , where overweight and obesity represented $85-97 \%$ and $>97 \%$, respectively, on age-differentiated percentile BMI charts (37). Obese, overweight and normal body mass in adult parents were determined according to BMI values (38). Overweight or obesity in parents represents a BMI from $25 \mathrm{~kg} / \mathrm{m}^{2}$ to $29.9 \mathrm{~kg} /$ $\mathrm{m}^{2}$ or greater than or equal to $30 \mathrm{~kg} / \mathrm{m}^{2}$, respectively (38). The chi-square test was used to compare the prevalence of obesity between sons and daughters (fathers and mothers). Daily step counts lower than 1,000 and higher than 30,000 were truncated to these recommended limit values (39) and included in the analysis. The variable of daily screen time represented the sum of 2 of the 7 items in the personalised individual logbook: sitting and lying while watching TV (DVD, video) and sitting and lying down in front of a PC (notebook, tablet, smartphone).

The data were analysed in total for all primary schools and both data collection rounds because the Two Step cluster analysis found no indicator for clustering by school or season. Means and $95 \%$ 
confidence intervals were computed for gender-specific steps/day and screen time/day for children and parents on weekdays and at weekends. Four 2-way (day of the week and gender) analyses of variance (ANOVA) were conducted to examine the differences between the days of the week (weekdays versus weekends) and gender (male versus female) effects on step counts (screen time) separately for children and parents. Weekdays and weekends were used as dependent variables to thoroughly examine gender effects on step counts (screen time) during the days of the week. Subsequently, to identify the differences in step counts (screen time) between weekdays/weekends in children and parents of both genders, Fisher's LSD post hoc test was used. The estimate of the strength of the relationship between the independent and dependent variables was represented as $\omega^{2}$ coefficient (40), where the values of $\omega^{2}<0.01,0.06-0.08$ and $0.14-0.18$ were interpreted as small, medium and large effect sizes, respectively (41). Several linear regressions were conducted to examine the association between parents' step counts/screen time and children's step counts on weekdays and at weekends stratified by gender.

\section{Ethics}

The study was approved by the Ethical Committee of the Faculty of Physical Culture, Palacký University in Olomouc. The children's parents, their teachers and school management representatives were informed in detail about the design of the survey during a joint class meeting at each of the participating schools. Written informed consent was obtained from the children's parents. All children and their parents participated in the study voluntarily and received no incentives. All study participants (children and parents) were provided with individual feedback on the results of the monitoring.

\section{RESULTS}

In total, 485 children (248 daughters and 237 sons) and 388 parents (245 mothers and 143 fathers) were eligible for this analysis. The mean values (standard deviations) or percentages of the children's and parents' anthropometric characteristics are presented in Table 1. Among all participating children we found significantly $(\mathrm{p}<0.05)$ higher percentages of obesity in sons $(12.34 \%)$ than in daughters $(6.43 \%)$. There was no significant difference in the prevalence of obesity in parents, however, a chi-square test revealed a significantly lower number $(\mathrm{p}<0.01)$ of mothers classified as overweight $(24.68 \%)$ compared with overweight fathers $(56.43 \%)$. The representation of overweight and obesity in parents and their children corresponded to the total prevalence of overweight and obesity in adults aged 35-44 years and children aged 9-12 years in the Czech Republic $(2,42)$.

The description of gender-related differences in the participants' step counts on weekdays and at weekends clearly indicates a lower number of steps on weekend days than on weekdays both in parents and their children (Fig. 1). Two-way ANOVAs uncovered significant differences in the step counts between weekdays and weekends in parents $\left(\mathrm{F}(2,766)=19.28, \mathrm{p}<0.001, \omega^{2}=0.045\right)$ and their children $\left(\mathrm{F}(2,970)=15.26, \mathrm{p}<0.001, \omega^{2}=0.028\right)$. Gender had a significant effect on step counts only in children $\left(F(1,970)=13.80, p<0.001, \omega^{2}=0.025\right)$. No interaction effects of gender and day of the week on the daily step counts of parents or their children were found.

Following the comparison of the differences in screen time (Fig. 2), considerable differences were revealed in the duration of screen time between weekdays and weekends in parents ( $\mathrm{F}$ $\left.(2,766)=4.20, \mathrm{p}<0.05, \omega^{2}=0.007\right)$ and their children $(\mathrm{F}(2$, $\left.970)=15.26, p<0.001, \omega^{2}=0.069\right)$. Gender had a significant effect on the duration of screen time only in parents $(\mathrm{F}(1,766)=27.33$, $\left.\mathrm{p}<0.001, \omega^{2}=0.063\right)$. No interaction effects of gender and day of the week on the duration of screen time of parents or their children were found.

The results relating to the quantification of the relationship between parents' step counts/screen time and children's step counts revealed a significant association both on weekdays (Fig. 3) and weekend days (Fig. 4). Each 1,000 step count increase in

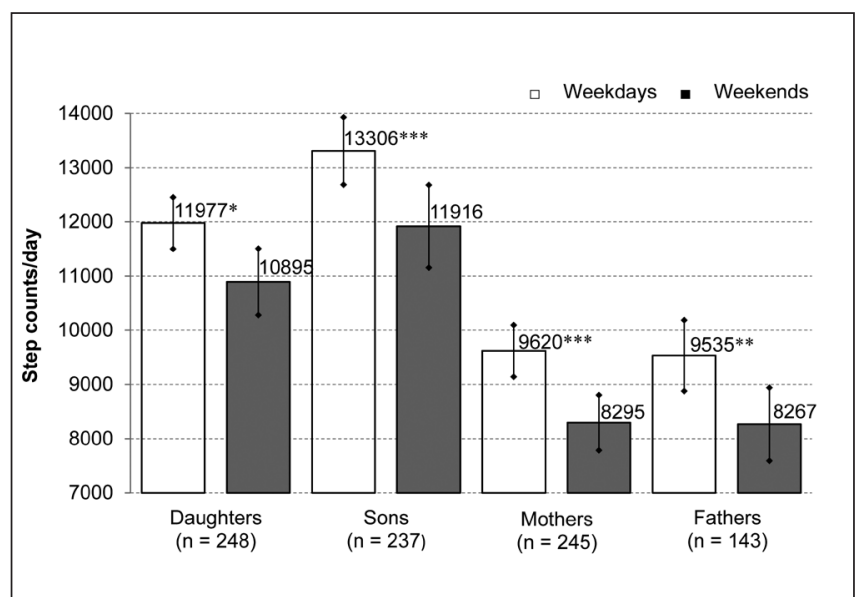

Fig. 1. Comparison of parents' and children's pedometerdetermined daily step counts (mean and 95\% confidence intervals) on weekdays and weekend days separated by gender; $n$-number of participants. The statistical significance (2-way analysis of variance, Fisher's LSD post hoc test) of the differences between weekday and weekend step counts is expressed as ${ }^{*} p<0.05,{ }^{* *} p<0.01$ and ${ }^{* * *} p<0.005$.

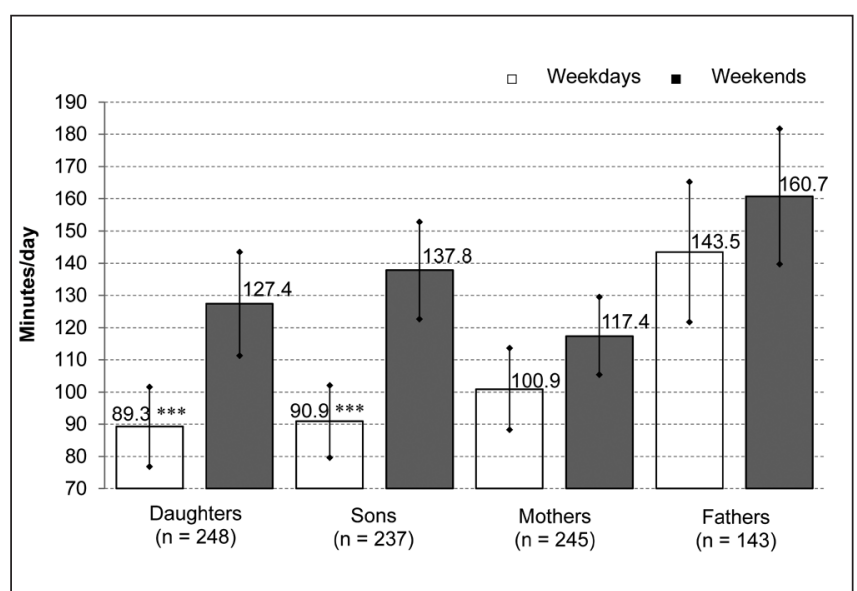

Fig. 2. Comparison of parents' and children's logged daily screen time (mean and 95\% confidence intervals) on weekdays and weekend days separated by gender; $n$ - number of participants. The statistical significance (2-way analysis of variance, Fisher's LSD post hoc test) of the differences between weekday and weekend minutes of screen time is expressed as ${ }^{* * *} p<0.005$. 
mothers' steps/weekday was associated with a significant increase by 261 daily steps $(\mathrm{p}<0.005)$ in their daughters and by 413 daily steps $(p<0.001)$ in their sons (Fig. 3). Every 1,000 step count increase in fathers' steps/weekday was linked to a significant increase by 244 daily steps $(\mathrm{p}<0.05)$ in their sons (Fig. 3$)$. The relationship between fathers' steps/weekday and daughters' steps/ weekday was not significant. At weekends a closer relationship between parents' and their children's step counts was uncovered compared with weekdays. Each 1,000 step count increase in mothers' steps/weekend day was associated with a significant increase by 523 steps $(\mathrm{p}<0.001)$ in their daughters and by 508 daily steps $(p<0.001)$ in their sons (Fig. 4). Moreover, every 1,000 increase in fathers' steps/weekend day was linked to a significant increase by 386 daily steps $(\mathrm{p}<0.005)$ in their daughters and by 435 daily steps $(\mathrm{p}<0.005)$ in their sons (Fig. 4).

In addition to the presented parent-child step count association, a significant relationship between the duration of weekend screen time in mothers and step counts in their children was found (Fig. 5). A reduction of screen time by 30 minutes on a weekend day was associated with a significant increase by 494 steps a day in their daughters $(\mathrm{p}<0.005)$ and by 467 steps a day in their sons $(\mathrm{p}<0.05)$.

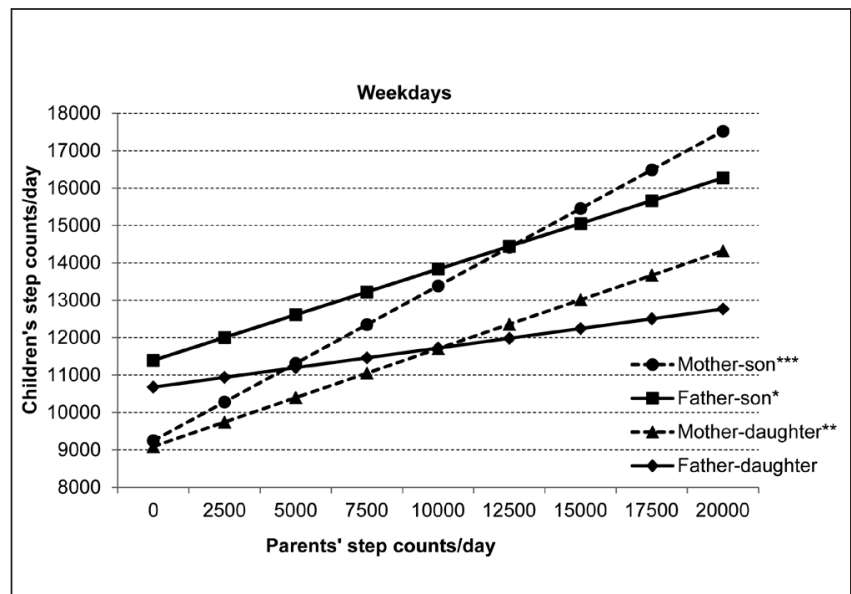

Fig. 3. Relationship between parents' and children's daily step counts (SC) on weekdays separated by gender. Statistical significance is expressed as ${ }^{*} p<0.05,{ }^{* *} p<0.005$ and ${ }^{* * *} p<0.001$.

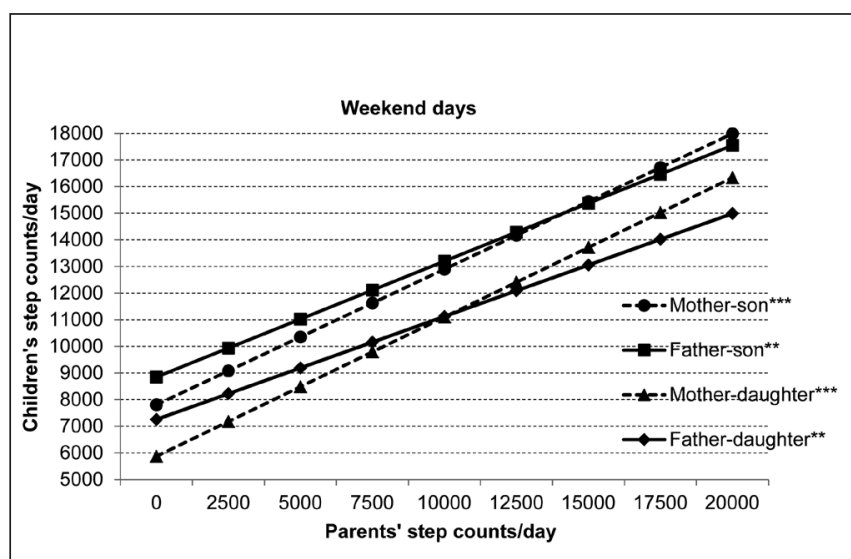

Fig. 4. Relationship between parents' and children's daily step counts (SC) on weekend days separated by gender. Statistical significance is expressed as ${ }^{* *} p<0.005$ and ${ }^{* * *} p<0.001$.

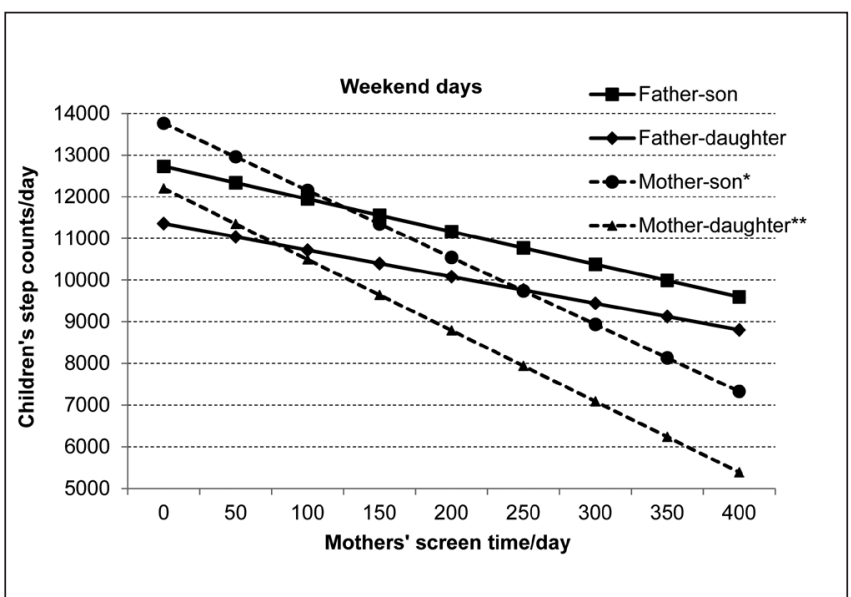

Fig. 5. Relationship between mothers' daily screen time (ST) and children's daily step counts (SC) on weekend days separated by gender. Statistical significance is expressed as ${ }^{*} p<0.05$ and ${ }^{* *} p<0.005$.

\section{DISCUSSION}

A positive link between objectively measured parent-child PA is known (7-9, 11, 21-24), so is the relationship between parent-child self-reported screen time $(25,26)$. However, the weekday-weekend variation of these relationships throughout a 7 -day continuous monitoring is still not well documented. The present study addresses these shortcomings by examining the gender-related association between parents' behaviour and children's step counts during a 7-day continuous monitoring period, separately on weekdays and weekend days.

In accordance with the first specific objective, gender-related differences in step counts and duration of screen time in parents and their children on weekdays and weekend days were identified. As in similarly designed studies $(9,23)$, and in line with a systematic literature review study (27), distinctly higher PA among children and parents of both genders on weekdays than on weekend days was found. However, a significant gender-related effect on step counts was revealed only in children. Boys (sons) achieved significantly more step counts per day than girls (daughters) on school days $(13,306 \pm 4,862$ vs. $11,977 \pm 3,804 \mathrm{p}<0.005)$ and on weekend days $(11,916 \pm 5,941$ vs. $10,895 \pm 4,903 \mathrm{p}<0.05)$. The dramatic difference in the step counts between weekdays and weekends is highlighted by the finding that on weekdays higher percentages of all participants $(43.6 \%$ of mothers and $38.5 \%$ of fathers, $55.6 \%$ of daughters and $49.8 \%$ of sons) met the current published daily step count recommendations (10,000 steps for adults (43), and 11,000 steps for girls and 13,000 steps for boys for primary school-aged children (44) than at weekends $(30.5 \%$ of mothers and $31.4 \%$ of fathers, $44.4 \%$ of daughters and $39.2 \%$ of sons).

The weekday-weekend patterns of screen time of parents and children were completely opposite to their weekday-weekend patterns of step counts. At weekends, parents and children of both genders showed a lower level of PA and increased screen time in comparison with weekdays/school days, which is confirmed by the results of other studies $(9,23,24,26)$. On weekdays, groups of daughters, sons and mothers, on average, did not reach 2 hours per day sitting or lying in front of the screen of a TV, PC 
or notebook, tablet or smartphone. However, at weekends, except for mothers, all other groups exceeded 2 hours of screen time per day. Furthermore, we discovered that a group of fathers showed the highest average daily screen time period, the lowest daily step counts and the highest proportion of overweight and obesity (68.6\%) among other family groups. Adult males in the Czech Republic are reported to have one of the highest proportions of overweight and obesity among Central and Eastern European men (1), and thus represent a serious public health problem.

For a better understanding of the effects on children's PA, recent studies call for clarification of the mechanisms by which both parents may influence their own children's PA $(8,9,11,22,23)$. In connection with the second specific objective, the relationship between parents' step counts/screen time and children's step counts on weekdays and weekends was quantified. A gender-stratified analysis of the relationship between parents' step counts/screen time and children's step counts revealed interesting findings that mothers' behaviour (step counts and screen time) is more positively associated with the PA of daughters and sons than fathers' behaviour, especially at weekends. This finding is consistent with a study unveiling a more pronounced influence of mothers on the PA of their children than fathers (45), but there are also studies that uncovered a 'gender-specific' behaviour tendency (i.e. mothers being correlated with daughters and fathers with sons) $(8,9)$, and even a study confirming a stronger association of fathers' PA on children's PA than mothers' PA (21). However, regardless of not entirely clear gender-related parent-child behaviour, a number of studies consistently indicate that children whose both parents are physically active are more likely to engage in PA than children of only one active parent or two sedentary parents $(8,9,21,45)$.

This study is one of the first surveys to investigate the differences in parent-child relationships concerning objectively measured PA separately for at least 4 weekdays/school days and 2 weekend days. As in a similarly designed study (8), a clear quantifiable gender-related relationship between parents' and children's step counts was discovered. However, in addition to a previous study (8), the parent-child step count relationship was quantified separately for weekdays and weekend days. In our study, each 1,000 step count increase in mothers' steps/weekday (steps/weekend day) was associated with an increase of 261 (523) daily steps in their daughters and 413 (508) daily steps in their sons, while every 1,000 step count increase in fathers' steps/weekday (steps/weekend day) was linked to an increase of 244 (435) daily steps in their sons and (386) in their daughters. Unlike the aforementioned study (8), we found a higher motherchild than father-child step count relationship on both weekdays and at weekends.

An area that previous research has not investigated is whether parental screen time is associated with the step counts of their children separately for weekdays and weekend days. While during weekdays/school days there was no significant relationship between parent screen time and the step counts of their children, at weekends a significant relationship between screen time in mothers and step counts in their children was revealed. Any reduction in mothers' screen time by 30 minutes per weekend day was associated with an increase by 494 (467) step counts per day in their daughters (sons). A replacement of 30 minutes of mothers' weekend screen time with joint PA with their children corresponds approximately to $31.6-48.9 \%(30.2-33.1 \%)$ of girls' (boys') step counts in a standard school physical education lesson or $46.1-67.9 \%$ (36.8-50.4\%) of girls' (boys') step counts during an unstructured school break (46).

In order to reach the recommended level $(11,000$ steps per day in girls and 13,000 steps per day in boys) of step counts in daughters (sons) during weekends it would be sufficient to increase the daily step counts by $201(2,129)$ in mothers or 272 $(2,491)$ in fathers or to reduce mothers' screen time by $6(67)$ minutes per weekend day (49). Nevertheless, this reasoning to increase children's PA at weekends through increased PA of parents (or reduced screen time among mothers) is based only on a generally accepted fact that parental influences are essential to the behaviour and PA of their children $(7-9,11,22,23)$. However, the aforementioned studies $(8,22)$ call for interventions to verify the direction of this relationship, evaluate the magnitude of influence, and elucidate the mediation and moderation of mechanisms by which both parents influence their children's PA.

\section{Strengths and Limitations}

The major strength of this study is the assessment of step counts (indicator of PA) as well as screen time (parameter of sedentary behaviour) for both mothers and fathers, which has facilitated the examination of maternal and parental associations with children's step counts. In contrast to other studies $(7-9,23-25)$, the strength of this one lies in the analysis of the parent-child relationship concerning step counts (relationship between parents' screen time and children's step counts) in participants who underwent complete monitoring for at least 10 hours a day during at least 4 weekdays/school days and 2 weekend days.

The findings of this study should be considered within the context of its limitations. Firstly, although the most widely used spring-levered pedometers provide an inexpensive valid and reliable assessment of total volume (step counts) of free-living PA, they are unable to accurately determine the pattern or intensity of PA (47). For more accurate monitoring of free-living PA and sedentary behaviour, the use of accelerometers (such as the ActiGraph, Actical or RT3 activity monitor) is necessary because accelerometers enable greater precision and accuracy than pedometers and are capable of an objective assessment of a 'minute-by-minute' pattern and intensity of PA as well as total volume of PA (47). Secondly, to minimize rejection of voluntary participation in the study, information about ethnicity, socioeconomic status, marital status, siblings, parents' job, parenting style and other family factors that might affect the PA behaviour of children was not investigated. Other factors that we have not examined in the study and that may affect PA and screen time of children include the influence of school teachers, coaches as well as classmates and peers. Neither the unquestionable effects of the environment of schools and residence on children's PA nor influence of the season or the weather were investigated in this study.

\section{CONCLUSIONS}

Uncovering the influences of parents' behaviour on their children's physical activity provides an insight into the lifestyle of families and developing effective family-based interventions. There is a clear quantifiable relationship between parents' and 
children's physical activity and parents' screen time and children's physical activity, especially at weekends. Every 1,000 step count increase in mothers' steps/weekend day (steps/weekday) was associated with an extra 523 (261) daily steps in their daughters and 508 (413) daily steps in their sons, while every 1,000 step count increase in fathers' steps/weekend day (steps/weekday) was linked to an increase of 435 (244) daily steps in their sons and 386 in their daughters. A reduction in mothers' screen time by 30 minutes per weekend day was associated with an increase of 494 (467) step counts per day in their daughters (sons). A replacement of at least 30 minutes of time that parents (especially mothers) and children spend together in sedentary pursuits with joint physical activity may result in increased weekend physical activity by a perceptible 500 steps per day.

\section{Acknowledgements}

This research and paper were funded by a project grant from the Czech Science Foundation (reg. No. GA16-14620S), and a research project from Palacký University Olomouc (IGA_FTK_2015_003). The founding party had no involvement in the data analysis, data interpretation or writing of the paper. The authors would like to thank all the children, parents and schools that participated in the study. Special thanks go to our parents (especially mothers), who have led us to physically active and healthy lifestyles since childhood.

\section{Conflict of Interest}

None declared

\section{REFERENCES}

1. Ng M, Fleming T, Robinson M, Thomson B, Graetz N, Margono C, et al. Global, regional, and national prevalence of overweight and obesity in children and adults during 1980-2013: a systematic analysis for the Global Burden of Disease Study 2013. Lancet. 2014 Aug 30;384(9945):766-81.

2. Sigmundová D, Sigmund E, Hamrik Z, Kalman M. Trends of overweight and obesity, physical activity and sedentary behaviour in Czech schoolchildren: HBSC study. Eur J Public Health. 2014 Apr;24(2):210-5.

3. Wijnhoven TM, van Raaij JM, Spinelli A, Starc G, Hassapidou M, Spiroski I, et al. WHO European Childhood Obesity Surveillance Initiative: body mass index and level of overweight among 6-9-year-old children from school year 2007/2008 to school year 2009/2010. BMC Public Health. 2014 Aug 7;14:806. doi: 10.1186/1471-2458-14-806.

4. Katzmarzyk PT, Barreira TV, Broyles ST, Champagne CM, Chaput JP, Fogelholm M, et al. The International Study of Childhood Obesity, Lifestyle and the Environment (ISCOLE): design and methods. BMC Public Health. 2013 Sep 30;13:900. doi: 10.1186/1471-2458-13-900.

5. Al-Hazzaa HM, Abahussain NA, Al-Sobayel HI, Qahwaji DM, Musaiger AO. Lifestyle factors associated with overweight and obesity among Saudi adolescents. BMC Public Health. 2012 May 16;12:354. doi: 10.1186/1471-2458-12-354.

6. Hills AP, King NA, Armstrong TP. The contribution of physical activity and sedentary behaviours to the growth and development of children and adolescents: implications for overweight and obesity. Sports Med. 2007;37(6):533-45

7. Jago R, Davison KK, Brockman R, Page AS, Thompson JL, Fox KR. Parenting styles, parenting practices, and physical activity in 10- to 11year olds. Prev Med. 2011 Jan;52(1):44-7.

8. Craig CL, Cameron C, Tudor-Locke C. Relationship between parent and child pedometer-determined physical activity: a sub-study of the CANPLAY surveillance study. Int J Behav Nutr Phys Act. 2013 Jan 18;10:8. doi: 10.1186/1479-5868-10-8.

9. Fuemmeler BF, Anderson CB, Mâsse LC. Parent-child relationship of directly measured physical activity. Int J Behav Nutr Phys Act. 2011 Mar 8;8:17. doi: 10.1186/1479-5868-8-17.

10. Rebholz CE, Chinapaw MJ, van Stralen MM, Bere E, Bringolf B, De Bourdeaudhuij I, et al. Agreement between parent and child report on parental practices regarding dietary, physical activity and sedentary behaviours: the ENERGY cross-sectional survey. BMC Public Health. 2014 Sep 5;14:918. doi: 10.1186/1471-2458-14-918.

11. McMinn AM, Griffin SJ, Jones AP, van Sluijs EM. Family and home influences on children's after-school and weekend physical activity. Eur J Public Health. 2013 Oct;23(5):805-10.

12. Sigmund E, El Ansari W, Sigmundová D. Does school-based physical activity decrease overweight and obesity in children aged 6-9 years? A two-year non-randomized longitudinal intervention study in the Czech Republic. BMC Public Health. 2012 Jul 29;12:570. doi: 10.1186/14712458-12-570.

13. Gortmaker SL, Peterson K, Wiecha J, Sobol AM, Dixit S, Fox MK, et al. Reducing obesity via a school-based interdisciplinary intervention among youth: Planet Health. Arch Pediatr Adolesc Med. 1999 Apr;153(4):409-18.

14. Kriemler S, Zahner L, Schindler C, Meyer U, Hartmann T, Hebestreit H, et al. Effect of school based physical activity programme (KISS) on fitness and adiposity in primary schoolchildren: cluster randomised controlled trial. BMJ. 2010 Feb 23;340:c785. doi: 10.1136/bmj.c785.

15. Sigmund E, Sigmundová D. Longitudinal 2-year follow-up on the effect of a non-randomised school-based physical activity intervention on reducing overweight and obesity of Czech children aged 10-12 years. Int J Environ Res Public Health. 2013 Aug 16;10(8):3667-83.

16. Vander Ploeg KA, Maximova K, McGavock J, Davis W, Veugelers P. Do school-based physical activity interventions increase or reduce inequalities in health? Soc Sci Med. 2014 Jul;112:80-7.

17. McAuley KA, Taylor RW, Farmer VL, Hansen P, Williams SM, Booker $\mathrm{CS}$, et al. Economic evaluation of a community-based obesity prevention program in children: the APPLE project. Obesity (Silver Spring). 2010 Jan;18(1):131-6.

18. Kothandan SK. School based interventions versus family based interventions in the treatment of childhood obesity - a systematic review. Arch Public Health. 2014 Jan 29;72(1):3. doi: 10.1186/2049-3258-72-3.

19. Van Lippevelde W, Verloigne M, De Bourdeaudhuij I, Brug J, Bjelland $\mathrm{M}$, Lien N, et al. Does parental involvement make a difference in schoolbased nutrition and physical activity interventions? A systematic review of randomized controlled trials. Int J Public Health. 2012 Aug;57(4):673-8.

20. Niemeier BS, Hektner JM, Enger KB. Parent participation in weightrelated health interventions for children and adolescents: a systematic review and meta-analysis. Prev Med. 2012 Jul;55(1):3-13.

21. Moore LL, Lombardi DA, White MJ, Campbell JL, Oliveria SA, Ellison RC. Influence of parents' physical activity levels on activity levels of young children. J Pediatr. 1991 Feb;118(2):215-9.

22. Hennessy E, Hughes SO, Goldberg JP, Hyatt RR, Economos CD. Parentchild interactions and objectively measured child physical activity: a cross-sectional study. Int J Behav Nutr Phys Act. 2010 Oct 7;7:71. doi: 10.1186/1479-5868-7-71.

23. Vander Ploeg KA, Kuhle S, Maximova K, McGavock J, Wu B, Veugelers PJ. The importance of parental beliefs and support for pedometermeasured physical activity on school days and weekend days among Canadian children. BMC Public Health. 2013 Dec 5;13:1132. doi: 10.1186/1471-2458-13-1132

24. Dunton GF, Liao Y, Almanza E, Jerrett M, Spruijt-Metz D, Chou CP, et al. Joint physical activity and sedentary behavior in parent-child pairs. Med Sci Sports Exerc. 2012 Aug;44(8):1473-80.

25. Jago R, Fox KR, Page AS, Brockman R, Thompson JL. Parent and child physical activity and sedentary time: do active parents foster active children? BMC Public Health. 2010 Apr 15;10:194. doi: 10.1186/14712458-10-194.

26. Jago R, Thompson JL, Sebire SJ, Wood L, Pool L, Zahra J, et al. Crosssectional associations between the screen-time of parents and young children: differences by parent and child gender and day of the week. Int J Behav Nutr Phys Act. 2014 Apr 23;11:54. doi: 10.1186/1479-5868-11-54.

27. Brooke HL, Corder K, Atkin AJ, van Sluijs EM. A systematic literature review with meta-analyses of within- and between-day differences in objectively measured physical activity in school-aged children. Sports Med. 2014 Oct;44(10):1427-38.

28. Jiménez-Pavón D, Konstabel K, Bergman P, Ahrens W, Pohlabeln H, Hadjigeorgiou C, et al.; IDEFICS consortium. Physical activity and clustered cardiovascular disease risk factors in young children: a crosssectional study (the IDEFICS study). BMC Med. 2013 Jul 30;11:172. doi: 10.1186/1741-7015-11-172.

29. Smrčková E, Chytil D. Population. In: Statistical yearbook of the Czech Republic 2013. Prague: Czech Statistical Office; 2013. p. 117-43.

30. Corder K, Ekelund U, Steele RM, Wareham NJ, Brage S. Assessment of physical activity in youth. J Appl Physiol (1985). 2008 Sep;105(3):977-87. 
31. Sigmundová D, Vašíčková J, Stelzer J, Řepka E. The influence of monitoring interval on data measurement: an analysis of step counts of university students. Int J Environ Res Public Health. 2013 Jan 28;10(2):515-27.

32. Sigmund E, Sigmundová D. School-related physical activity, lifestyle and obesity in children [Internet]. Olomouc: Palacký University in Olomouc; 2014 [cited 2015 Oct 30]. Available from: http://flexibooks.cz/schoolrelated-physical-activity-lifestyle-and-obesity-in-children. (In Czech.)

33. Tudor-Locke C, Ainsworth BE, Thompson RW, Matthews CE. Comparison of pedometer and accelerometer measures of free-living physical activity. Med Sci Sports Exerc. 2002 Dec;34(12):2045-51.

34. Crouter SE, Schneider PL, Karabulut M, Bassett DR Jr. Validity of 10 electronic pedometers for measuring steps, distance, and energy cost. Med Sci Sports Exerc. 2003 Aug;35(8):1455-60.

35. Cuberek R, El Ansari W, Frömel K, Skalik K, Sigmund E. A comparison of two motion sensors for the assessment of free-living physical activity of adolescents. Int J Environ Res Public Health. 2010 Apr;7(4):1558-76.

36. Chan NP, Choi KC, Nelson EA, Sung RY, Chan JC, Kong AP. Selfreported body weight and height: an assessment tool for identifying children with overweight/obesity status and cardiometabolic risk factors clustering. Matern Child Health J. 2013 Feb;17(2):282-91.

37. World Health Organization. WHO child growth standards: length/heightfor-age, weight-for-age, weight-for-length, weight-for-height and body mass index-for-age: methods and development [Internet]. Geneva: WHO; 2006 [cited 2015 Oct 30]. Available from: http://apps.who.int/iris/bitstre am/10665/43413/1/924154693X_eng.pdf?ua=1.

38. World Health Organization. Media centre. Obesity and overweight [Internet]. Geneva: WHO; 2014 [cited 2014 Aug 27]. Available from: http:// www.who.int/mediacentre/factsheets/fs311/en/.

39. Craig CL, Cameron C, Griffiths JM, Tudor-Locke C. Descriptive epidemiology of youth pedometer-determined physical activity: CANPLAY. Med Sci Sports Exerc. 2010 Sep;42(9):1639-43.
40. Cohen J. Statistical power analysis for the behavioral sciences. 2nd ed. New York, NY: Lawrence Erlbaum Associates; 1988.

41. Velicer WF, Cumming G, Fava JL, Rossi JS, Prochaska JO, Johnson J. Theory testing using quantitative predictions of effect size. Appl Psychol. 2008 Oct;57(4):589-608.

42. Sigmundová D, El Ansari W, Sigmund E. Neighbourhood environment correlates of physical activity: a study of eight Czech regional towns. Int J Environ Res Public Health. 2011 Feb;8(2):341-57.

43. Tudor-Locke C, Craig CL, Brown WJ, Clemes SA, De Cocker K, GilesCorti B, et al. How many steps/day are enough? For adults. Int J Behav Nutr Phys Act. 2011 Jul 28;8:79. doi: 10.1186/1479-5868-8-79.

44. Tudor-Locke C, Craig CL, Beets MW, Belton S, Cardon GM, Duncan S, et al. How many steps/day are enough? For children and adolescents. Int J Behav Nutr Phys Act. 2011 Jul 28;8:78. doi: 10.1186/1479-5868-8-78.

45. Griffith JR, Clasey JL, King JT, Gantz S, Kryscio RJ, Bada HS. Role of parents in determining children's physical activity. World J Pediatr. 2007;3(4):265-70.

46. Sigmund E, Sigmundová D, Šnoblová R, Gecková AM. ActiTrainerdetermined segmented moderate-to-vigorous physical activity patterns among normal-weight and overweight-to-obese Czech schoolchildren. Eur J Pediatr. 2014 Mar;173(3):321-9.

47. Rowlands AV, Eston RG. The Measurement and interpretation of children's physical activity. J Sports Sci Med. 2007 Sep 1;6(3):270-6.

Received November 13, 2014 Accepted in revised form September 16, 2015 